\title{
Hybrid Technique for Locating and Sizing of Renewable Energy Resources in Power System
}

\author{
M. Durairasan ${ }^{\dagger}$, A. Kalaiselvan* and H. Habeebullah Sait**
}

\begin{abstract}
In the paper, a hybrid technique is proposed for detecting the location and capacity of distributed generation (DG) sources like wind and photovoltaic (PV) in power system. The novelty of the proposed method is the combined performance of both the Biography Based Optimization (BBO) and Particle Swarm Optimization (PSO) techniques. The mentioned techniques are the optimization techniques, which are used for optimizing the optimum location and capacity of the DG sources for radial distribution network. Initially, the Artificial Neural Network (ANN) is applied to obtain the available capacity of DG sources like wind and PV for 24 hours. The BBO algorithm requires radial distribution network voltage, real and power loss for determining the optimum location and capacity of the DG. Here, the BBO input parameters are classified into sub parameters and allowed as the PSO algorithm optimization process. The PSO synthesis the problem and develops the sub solution with the help of sub parameters. The BBO migration and mutation process is applied for the sub solution of PSO for identifying the optimum location and capacity of DG. For the analysis of the proposed method, the test case is considered. The IEEE standard bench mark 33 bus system is utilized for analyzing the effectiveness of the proposed method. Then the proposed technique is implemented in the MATLAB/simulink platform and the effectiveness is analyzed by comparing it with the $\mathrm{BBO}$ and PSO techniques. The comparison results demonstrate the superiority of the proposed approach and confirm its potential to solve the problem
\end{abstract}

Key words: DG, Wind, PV, BBO, PSO, Voltage, Power and power loss

\section{Introduction}

Recently, Distributed Generations (DGs) tend to be taking part in an important purpose in Electrical Distribution Systems (EDS) due to ecological issues, fuel charge uncertainties along with liberalization associated with energy marketplaces [1]. DGs tend to be small-scale bringing in devices between numbers of KWs for you to 100 MWs: micro, small, medium and huge DGs, which are fitted at the load facilities to attenuate power deficits along with inefficiencies [2]. Compared to huge main power plants based on fossil fuel, oil, along with gas-fired plants [3], DGs resources for instance wind generators, photovoltaic, fuel cells, biomass, micro generators, small hydroelectric plant, landfill gas, and so on. [4] get a reduced amount of funds expenditure along with maintenance and upkeep costs, the area is a lot easier to get and possess less unfavorable affect the surroundings [5]. The important thing advantages which is available from DGs tend to be advancement associated with voltage stability \& voltage profile, power

$\dagger \quad$ Department of Electrical and Electronics Engineering, University College of Engineering, Anna University, Thirukkuvalai, India. (durairasan0104@gmail.com)

* Department of Chemistry, University College of Engineering, Anna University, Thirukkuvalai, India. (kalai.anba@gmail.com)

** Department of Electrical and Electronics Engineering, BIT Campus, Anna University, Tiruchirappalli, India.

Received: March 16, 2016; Accepted: July 18, 2016 loss lessening, superior system trustworthiness \& safety along with improved power quality (PQ) \& service associated with additional solutions [6-7]. It's also very helpful in cutting down the output price. DGs can be utilized possibly in a separated way or even in an incorporated way. As a result, it can present in the locations where the central generation can be impracticable or even wherever you can find deficiencies in the actual transmitting process [8-9].

Within EDS, DGs might help intended for theoretically and financially because, they are able to postponement connected with transmission and distribution lines upgrade ventures [10]. DGs have a considerable effect on the power movement, voltage profile, stability, continuity, short circuit level, and high quality connected with power intended for clients and energy consumers [11-12]. The actual installation connected with DGs may possibly best to network system procedure; however too much amount or even dimensions connected with DGs in operation might cause violations in the technique limitations [13]. That's why; the correct sizes and placement connected with distributed turbines is essential intended for maximum benefit in the electrical power system [14]. In the event DG products tend to be incorporated on non-optimal spots, the power deficits increase, leading to elevated expense of power, voltage increase at the end of your feeder, need supply unbalance within a fault situation, electrical power high quality drop and decrease regarding reliability ranges 
[15]. A few other concerns associated the actual DG installation tend to be harmonic shots, the call to embrace more advanced command plans, and the possibility of discovering slow electrical power flows in electrical power networks and the related dangers to help linemen safety [16]. The choice connected with optimum sizes and sitting is founded on loss minimization, enhancement in the voltage profile, and decrease in the electrical power flows over the lines, lowering expenditure and procedure prices, and many others. [17-18]. Therefore, the perfect offering of DGs in EDS is a complex combinatorial marketing issue [19]. Within literary works, a number of methods provide: classical optimizations, analytical methods, meta-heuristics and hybrid methods and so on. [20].

A hybrid technique has been discussed in this paper for location and the capacity of distribution generator (DG) in power system. The novelty of the proposed method is the combined performance of both the Biography Based optimization (BBO) and Particle Swarm Optimization (PSO) techniques.

\section{Recent Related Works}

Partha Kayal et al. [21] have proposed a constrained multi-objective Particle Swarm Optimization (PSO) based Wind Turbine Generation Unit (WTGU) and photovoltaic (PV) array placement approach for power loss reduction and voltage stability improvement of radial distribution system. The paper reflects the effectiveness of WTGU and PV array performance models in DG placement problem formulation. Wind and solar based DGs were operated in different active and reactive power mode and tested on 12bus, 15-bus, and 33-bus and 69-bus radial distribution system. Obtained results were compared with other DG placement technique and proposed method was found to be more effective in terms of voltage stability enhancement and power loss minimization. A Voltage Stability Factor (VSF) has been proposed in their paper which can quantify voltage stability levels of buses in the system. Comparing with other voltage stability index and power stability index, developed VSF has emerged as more simple and efficient tool.

M.M. Aman et al. [22] have presented an approach for optimum simultaneous multi-DG (distributed generation) placement and sizing based on maximization of system loadability without violating the system constraints. DG penetration level, line limit and voltage magnitudes were considered as system constraints. HPSO (hybrid particle swarm optimization) algorithm was also proposed in their paper to find the optimum solution considering maximization of system loadability and the corresponding minimum power losses. The proposed method was tested on standard 16-bus, 33-bus and 69-bus radial distribution test systems. Their paper also compare the proposed method with existing Ettehadi method and present the effectiveness of the proposed method in terms of reduction in power system losses, maximization of system loadability and voltage quality improvement.

Zhaoyu Wang et al. [23] have proposed a Microgrid (MG) planning methodology to decide optimal locations, sizes and mix of dispatchable and intermittent distributed generators (DGs). The long-term costs in the proposed planning model include investment, operation and maintenance (O\&M), fuel and emission costs of DGs while the revenue includes payment by MG loads and utility grid. The problem was formulated as a mixed-integer program (MIP) considering the probabilistic nature of DG outputs and load consumption, wherein the costs were minimized and profits are maximized. The model was transformed to be a two-stage robust optimization problem. A column and constraint generation (CCG) framework was used to solve the problem. Compared with conventional MG planning approaches, the proposed model is more practical in that it fully considers the system uncertainties and only requires a deterministic uncertainty set, rather than a probability distribution of uncertain data which was difficult to obtain. Case studies of a MG with wind turbines, photovoltaic generators (PVs) and micro turbines (MTs) demonstrate the effectiveness of their proposed methodology.

Srinivasa Rao Gampa et al. [24] have presented a multi objective technique for obtaining optimal sizing of Distributed Generation (DG) units considering both technical and economical factors of the distribution system. The technical factors include real power loss reduction, line load reduction and voltage profile improvement and the economical factors consider optimal DG investment cost. Three different Distributed Generation systems solar photovoltaic, biomass and wind system were considered for integration with the existing distribution system. Since solar photovoltaic system was not available at night time, only biomass and wind systems were operated and for day time operation all the three distribution generation systems were considered. A sensitivity index based on voltage sensitivity and apparent load power was proposed for identification of optimal locations for DG placement. The optimum sizing of DG units operating at unity power factor and lagging power factor was obtained using GA for different load levels considering daily average hourly loading aiming at improving the technical performance of the distribution system with optimum investment on DG units.

Ke-yan Liu et al. [25] have presented a method for optimal sitting and sizing of DG in distributed system with the consideration of time sequence characteristics of load and distributed generator (DG) output. Multi-objective functions have been formulated with the consideration of minimum investment and operational cost of DG, minimum purchasing electricity cost from main grid and minimum voltage deviation. To solve the multi-objective optimization problem, an improved Non-dominated Sorting Genetic Algorithm II has been proposed. The compromised 
solution was extracted from the Pareto set using the fuzzy theory method. Several experiments have been made on the modified PG\&E 69-bus and multiple actual test cases with the consideration of multiple DGs. The computational result and comparisons indicate the proposed method for optimal placement and sizing of DG was feasible and effective.

Rajkumar Viral et al. [26] have presented an analytical approach to determine the optimal setting and sizing of distributed generation (DG) units in balanced radial distribution network to minimize the power loss of the system. Their proposed analytical expressions were based on a minimizing the loss associated with the active and reactive component of branch currents by placing the DG at various locations. Their method first identifies a sequence of nodes where DG units were to be placed. The optimal sizes of DG units at the identified nodes were then evaluated by optimizing the loss saving equations and need only the results of base case load flow. To find out the best location for DG placement, a computational method was also developed. The proposed method has been tested and validated on two IEEE test distribution systems (DSs) consisting of 15 and 33-buses and it has been found that a significant loss saving can be obtained by placing DG units in the system using their proposed analytical method.

Partha Kayal et al. [27] have presented a simple efficient approach for optimal placement and sizing of solar and wind DGs in distribution territory by considering electrical network power loss minimization, voltage stability and network security improvement. The stochastic nature of solar irradiance and wind speed were accounted using suitable probabilistic models. Weighted aggregation particle swarm optimization technique was employed to optimize the objective functions considering bus voltage limit, line loading capacity, discrete size limit and penetration constraints of DGs. Strategic weight selection technique has been adopted to assess the well trade-off solution by persuasion of multiple objectives regarding the performance of distribution network. Their proposed method has been applied to a typical Indian rural distribution network, and the satisfactory results were obtained.

\section{Problem Formulation}

The DG units' installation at optimal location will lead to line loss reduction, improved voltage stability, reliability and security. In the paper, the DG units are considered as the wind and PV respectively. The optimum location and sizing of the DG is the optimization problem with nonlinear objective function with the corresponding constraints like power balance constraint, voltage constraint and DG constraints etc. The main aim of the proposed method is that, it should reduce the exact power loss, load balancing and voltage deviation of the given radial distribution network at the peak load condition. Hence, it utilizes the multi-objective function to determine the optimum location and sizing of the DG. Here, the multiobjective function is mathematically formulated as the following Eq. (1).

$$
\Phi=\operatorname{Min}\left\{f_{1}, f_{2}, f_{3}\right\}
$$

where, $f_{1}, f_{2}$ and $f_{3}$ are the power loss, voltage deviation and load balancing respectively. The mathematical equation of the multi-objective function is described as follows:

\subsection{Power loss $\left(f_{1}\right)$}

The distribution systems build instability during the peak loading conditions, which leads to constraint violations. The obtained DG location and capacity should minimize the power loss and realizes the limits of the constraints. The required exact loss of the distribution system is calculated by the following Eq. (2).

$$
f_{1}=P L^{D}=\sum_{i=1}^{N} \sum_{j=1}^{N}\left[\alpha_{i j}\left(P_{i} P_{j}+Q_{i} Q_{j}\right)+\beta_{i j}\left(Q_{i} P_{j}-P_{i} Q_{j}\right)\right]
$$

where, $\alpha_{i j}=\frac{r_{i j}}{V_{i} V_{j}} \cos \left(\delta_{i}-\delta_{j}\right), \quad \beta_{i j}=\frac{r_{i j}}{V_{i} V_{j}} \sin \left(\delta_{i}-\delta_{j}\right)$

With $P L^{D}$ is the exact loss of the distribution system; $r_{i j}$ is the resistance between bus $i$ and bus $j ; V_{i}$ and $V_{j}$ is the voltage magnitude of buses $i$ and $j$ respectively; $\delta_{i}$ is the voltage angle at bus $i ; \delta_{j}$ is the voltage angle at bus $j ; P_{i}$ and $Q_{i}$ active and reactive power injection at bus $i ; P_{j}$ and $Q_{j}$ is the active and reactive power injection at bus $j$. The voltage deviation is determined by the following section 3.2.

\subsection{Voltage deviation $\left(f_{2}\right)$}

The radial distribution network voltage profile management is a main factor. When DG is connected to a distribution network, the voltage profile is changed. It can be evaluated at all of the buses in the radial distribution systems. Here, the identified DG should minimize the difference between the normal bus voltage and the specified bus voltage for improving the voltage stability. The required voltage deviation equation is described in the following Eq. (5).

$$
f_{2}=\sum_{i=1}^{N}\left(\frac{V_{i}-V_{\text {spec }}}{V_{\text {spec }}}\right)
$$

where, $V_{\text {spec }}$ is the specified voltage; normally the specified voltage is taken between 0.95 to 1.05 limit. The objective load balancing equation is described in the 
following section 3.3 .

\subsection{Load balancing $\left(f_{3}\right)$}

Here, the following Eq. (6) represents the load balancing of the lines.

$$
f_{3}=\sum_{i=1}^{N}\left(\frac{I_{i}}{I_{L}^{\text {avg }}}\right)
$$

where, $I_{i}$ is current of branch $i$; this branch current should not exceed the thermal limit, i.e., $I_{i} \leq I_{i}^{\text {rated }}, I_{N}^{\text {avg }}$ is the average current of the total lines, which is determined by the following relation (7).

$$
I_{N}^{a v g}=\sum_{L=1}^{N}\left(\frac{I_{L}}{N}\right)
$$

where, $N$ is the total number of lines; the subjected constraints and the mathematical equations, which are explained in the following section 3.4.

\subsection{Constraints}

\subsubsection{Power balance constraint}

The power balance equation mainly describes about the generated DG power must satisfy the

$$
\begin{aligned}
& P_{D i}^{D}=P_{G i}^{D}-Y_{i j} \sum_{i=j=1}^{N} V_{i} V_{j} \cos \left(\delta_{i}-\delta_{j}-\theta_{i}\right) \\
& Q_{D i}^{D}=Q_{G i}^{D}-Y_{i j} \sum_{i=j=1}^{N} V_{i} V_{j} \sin \left(\delta_{i}-\delta_{j}-\theta_{i}\right)
\end{aligned}
$$

where, $P_{G i}^{D}$ and $Q_{G i}^{D}$ is the power generations of generators at bus $i ; Y_{i j}$ is the admittance of the line between $i$ and $j ; \theta_{i}$ is phase angle of the bus $i ; P_{D i}^{D}$ and $Q_{D i}^{D}$ is the load demand at bus $j$; which is lies in the following limit $P_{D i}^{D(\min )} \leq P_{D i}^{D} \leq P_{D i}^{D(\max )}$ and $Q_{D i}^{D(\min )} \leq Q_{D i}^{D} \leq Q_{D i}^{D(\max )}$.

\subsubsection{Voltage constraint}

The voltage limits of each bus must lie within the prescribed limits, which are given in the following Eq. (10).

$$
V^{\min } \leq V_{i} \leq V^{\max }
$$

where, $V^{\min }$ and $V^{\max }$ are the minimum and maximum values of voltage at bus $i$; normally the bus voltage lies between $0.95 \leq V_{i} \leq 1.05 \mathrm{pu}$.

\subsubsection{DG constraint}

The DG constraint mainly consists of the allowable capacity for the buses and the corresponding power factor of the DG. The capacity of the DG is described in the following Eq. (11).

$$
\begin{gathered}
\sum_{i \in N} \sqrt{\left(P_{i}^{D G}\right)^{2}+\left(Q_{i}^{D G}\right)^{2}} \leq \frac{1}{2} \sum_{i \in N} \sqrt{\left(P_{i}^{L}\right)^{2}+\left(Q_{i}^{L}\right)^{2}} \\
P F_{\min }^{D G} \leq P F_{i}^{D G} \leq P F_{\max }^{D G}
\end{gathered}
$$

where, $P F_{\min }^{D G}$ and $P F_{\max }^{D G}$ is the minimum and maximum values of power factor at the DG bus $i$. The proposed method utilizes the constraints to find the location, which has more priority of real power loss sensitivity factor. Depending on the loss function the corresponding capacity of DG has been identified and added to the particular location. The brief process about the determination of DG location and capacity using the proposed method is described in the following section 4 .

\section{Hybrid Technique for Optimum Location and Capacity of DG}

The proposed hybrid technique has the combined operation of both the BBO algorithm and PSO algorithm. The mentioned techniques are the optimization techniques, which are used for optimizing the optimum location and capacity of the DG for radial distribution network. The $\mathrm{BBO}$ algorithm requires radial distribution network voltage, real and reactive power for the optimum location and capacity of the DG. Here, the input parameters are spitted into sub parameters and allowed as the PSO algorithm. The PSO synthesis the problem and develops the sub solution with the help of sub parameters. The BBO migration and mutation process is applied for the sub solution of PSO and identify the optimum location and capacity of DG. Before that, the ANN is applied for obtained the DG capacity for 24 hours. Then the best solution is finalized from both techniques results. The detailed description of the ANN is represented in the following section.

\subsection{Utilizing ANN to find the DG capacity}

Initially, the optimal location of the DG sources like wind and PV is determined based on their availability. Here, the test case is considered as the IEEE standard bench mark 33 bus system. For 24 hours, the availability of DG sources is evaluated using $\mathrm{ANN}$, which provides the available minimum and maximum limits. Here, we can use the hourly DG capacity dataset in a day. The hourly DG capacity dataset is used to train the ANN.

\subsection{Artificial neural network}

The ANN technique works on the basis of a machine learning approach that models a human brain and consists 


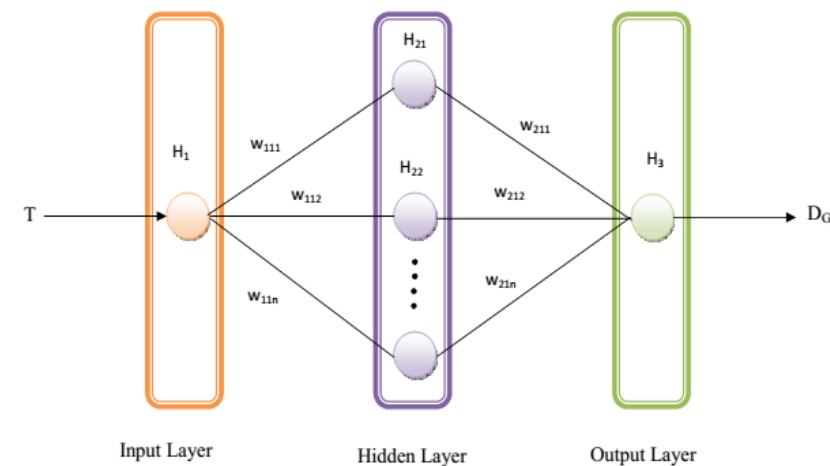

Fig. 1. Structure of the ANN

of a number of artificial neurons. The presented neurons have the interior connections and each neuron in ANN receives a number of inputs, depending on the activation functions of the ANN results in the output level of the neuron. The learning task is given in the form of examples, which is known as training examples [28]. Generally the ANN structure consists of three layers like input layer, hidden layer and output layer, which is explained in the Fig. 1. Here, we are training the ANN using the target capacity with the corresponding input time intervals of a day dataset. The back propagation algorithm is utilized for the training process. The training process is explained in the following.

\section{Back propagation learning algorithm steps:}

Step 1: Initialization of the input layer, hidden layer and output layer weights of the neural network. Here, the time interval $T$ is the input of the network, DG capacity $D_{g}(t)$ is the output of the network.

Step 2: Learning the network according to the input and the corresponding target.

Step 3: Calculate the back propagation error of the target $D_{g}(1), D_{g}(2)$ and $D_{g}(n)$.

$$
\left.\begin{array}{l}
B P_{\text {error }}^{1}=D_{g}(1)^{N N(\text { tar })}-D_{g}(1)^{N N(\text { out })} \\
B P_{\text {error }}^{2}=D_{g}(2)^{N N(\text { tar })}-D_{g}(2)^{N N(\text { out })} \\
B P_{\text {error }}^{n}=D_{g}(n)^{N N(\text { tar })}-D_{g}(n)^{N N(\text { out })}
\end{array}\right\}
$$

where, $D_{g}(n)^{N N(t a r)}$ is the network target of the $n^{\text {th }}$ node and $D_{g}(n)^{N N(\text { out })}$ is the current output of the network.

Step 4: The output of the network is determined by following them,

$$
\left.\begin{array}{l}
D_{g}(1)^{N N(\text { out })}=\alpha_{1}+\sum_{n=1}^{N} w_{1 n} D_{g}(1)^{N N(k)} \\
D_{g}(2)^{N N(\text { out })}=\alpha_{2}+\sum_{n=1}^{N} w_{1 n} D_{g}(2)^{N N(k)} \\
D_{g}(n)^{N N(\text { out })}=\alpha_{n}+\sum_{n=1}^{N} w_{1 n} D_{g}(n)^{N N(k)}
\end{array}\right\}
$$

where, $\alpha_{1}, \alpha_{2}$ and $\alpha_{n}$ are the bias function of the node 1,2 and $n$ respectively.

$$
\left.\begin{array}{l}
D_{g}(1)^{N N(k)}=\frac{1}{1+\exp \left(-w_{1 n} D_{g}(1)-w_{2 n} D_{g}(2)\right)} \\
D_{g}(2)^{N N(k)}=\frac{1}{1+\exp \left(-w_{1 n} D_{g}(2)-w_{2 n} D_{g}(n)\right)} \\
D_{g}(n)^{N N(k)}=\frac{1}{1+\exp \left(-w_{1 n} D_{g}(n)-w_{2 n} D_{g}(1)\right)}
\end{array}\right\}
$$

Step 5: The new weights of the each neurons of the network are updated by $w_{\text {new }}=w_{\text {old }}+\Delta w$. Here, $w_{\text {new }}$ is the new weight, $w_{\text {old }}$ is the previous weight and $\Delta w$ is the change of weight of each output. The change of weight is determined as follows:

$$
\left.\begin{array}{rl}
\Delta w_{1} & =\delta \cdot D_{g}(1) \cdot B P_{\text {error }}^{1} \\
\Delta w_{2} & =\delta \cdot D_{g}(2) \cdot B P_{\text {error }}^{2} \\
\Delta w_{k} & =\delta \cdot D_{g}(n) \cdot B P_{\text {error }}^{n}
\end{array}\right\}
$$

where, $\delta$ is the learning rate (0.2 to 0.5$)$.

Step 6: Repeat the above steps till the $B P_{\text {error }}$ gets minimized $B P_{\text {error }}<0.1$.

Once the neural network training process is completed, the network is trained well for the identifying DG capacity according to the input time interval. Based on the output of the network, the hybrid BBO and PSO algorithm optimizes the optimal location. The next process is the optimum location finding, which can be done by using the hybrid BBO-PSO technique. It is briefly explained in section 4.2.

\subsection{BBO and PSO algorithm for optimal location and capacity of $D G$}

$\mathrm{BBO}$ is a population based, stochastic optimization technique, which is based on the concept of biogeography that deals with nature's way of distribution of species. Distribution of a species from one place to another is influenced by factors such as rainfall, diversity of vegetation, diversity of topographic features, land area, temperature etc. Areas, where these factors are highly favorable tend to have a larger number of species, compared with a less favorable area. Movement of species from one area to another area facilitates sharing of their features with each other [29]. Owing to this movement, the quality of some species may improve due to exchange of good features with better species. In context of biogeography, a habitat is defined as an Island (area) that is geographically isolated from other Islands. Geographical areas that are well suited as residences for biological species are said to have a high habitat suitability index (HSI). The variables that characterize habitability are called suitability index variables (SIVs). SIVs can be considered as the independent 
variables of the habitat and HSI calculation is carried out using these variables [30]. The migration of some species from a habitat to an exterior habitat is known as emigration process and an entry into one habitat from an outside is known as immigration process. The rate of immigration and the emigration are functions of the number of species in the habitat. Habitats with a high HSI have a low species immigration rate as they are already saturated with species. In the paper, $\mathrm{BBO}$ algorithm is utilized for detecting the optimal location of DG sources like wind and PV.

Here, the steps to identify the optimum location and capacity of DG for radial distribution network are described in the following.

\section{Steps to find the optimum location and capacity of DG}

Step 1: Initialize the BBO input parameters like radial distribution network bus voltage $\left(V_{i}\right)$, real and reactive power $\left(P_{i}\right.$ and $\left.Q_{i}\right)$ etc.

Step 2: Run the load flow of the radial distribution network for normal condition.

Step 3: Find the normal condition power loss using the Eq. (2) and identify the real power loss sensitivity factor using the following Eq. (13) for all the buses. Arrange the bus loss sensitivity factor as descending order.

$$
R P_{l s f}=\frac{\Delta P L^{D}}{\Delta P_{i}}=2 \sum_{i=1}^{N}\left(\alpha_{i j} P_{j}-\beta_{i j} Q_{j}\right)
$$

Step 4: Using the multi-objective function (1), the BBO identifies the optimum location and capacity of DG.

Step 5: The input BBO input parameters are classified into sub parameters and used as the PSO initialization. The PSO input parameters classification is explained in the following Fig. 2.

Step 6: The PSO randomly generates the ' $N$ ' number of initial populations. In the paper radial distribution network bus voltage $\left(V_{i}\right)$, real and reactive power $\left(P_{i}\right.$ and $\left.Q_{i}\right)$ is selected as an initial population.

Step 7: For each particle find the real power sensitivity factor (13) and arrange the bus loss sensitivity factor as descending order. In each particle, the fitness has been evaluated by using the power loss, voltage deviation and load balancing equations.

Step 8: Depending on the fitness function evaluation the particles are classified into two groups, i.e., maximum fitness and minimum fitness. From the classification, the optimum location and capacity of DG is determined as per the multi-objective function (1).

Step 9: For each best particle velocity and position are modified by using the following Eq. (14) and (15).

$$
\begin{gathered}
v[]=v[]+c 1 * \operatorname{rand}() *(\operatorname{pbest}[]-\operatorname{present}[]) \\
+c 2 * \operatorname{rand}() *(\operatorname{gbest}[]-\operatorname{present}[]) \\
\operatorname{present}[]=\operatorname{present}[]+v[]
\end{gathered}
$$

$v[$ ] is the particle velocity, present [ ] is the current particle, pbest[ ] and gbest[ ] are best fitness value and best value from any particle in the population respectively, $\operatorname{rand}()$ is the random number between $(0,1)$ and $c 1, c 2$ are learning factors [31,32].

Step 10: Using the updated particles again evaluate the multi-objective function.

Step 11: Check the iteration limit, if it reaches the maximum number, go to or else increase the iteration number and go back to the step 7 .

Step 12: Finalize the best solution by comparing the PSO result with the $\mathrm{BBO}$ result.

Step 13: Terminate the process.

Once the process is finished the proposed mutual technique is ready to give the best location and capacity of the DG for radial distribution network. Then the overview of the proposed method is described in the following Fig. 2. The optimum location and capacity of DG determination using the PSO by utilizing the BBO input parameter is explained in Fig. 3. Then the proposed mutual technique is tested under MATLAB platform through the IEEE standard benchmark system. The tested results are compared with the other techniques like BBO and PSO. The proposed mutual technique results and corresponding discussion is given in the following section 5 .

\section{Results and Discussion}

In the paper, the proposed method is implemented in MATLAB/simulink platform, 4GB RAM and Intel(R) core(TM) i5. By utilizing the proposed method, the optimal location of DG sources is determined and their sizing also evaluated. The Wind and Photovoltaic (PV) are considered



Fig. 2. PSO input parameters classification 




Fig. 3. Structure of the proposed hybrid technique

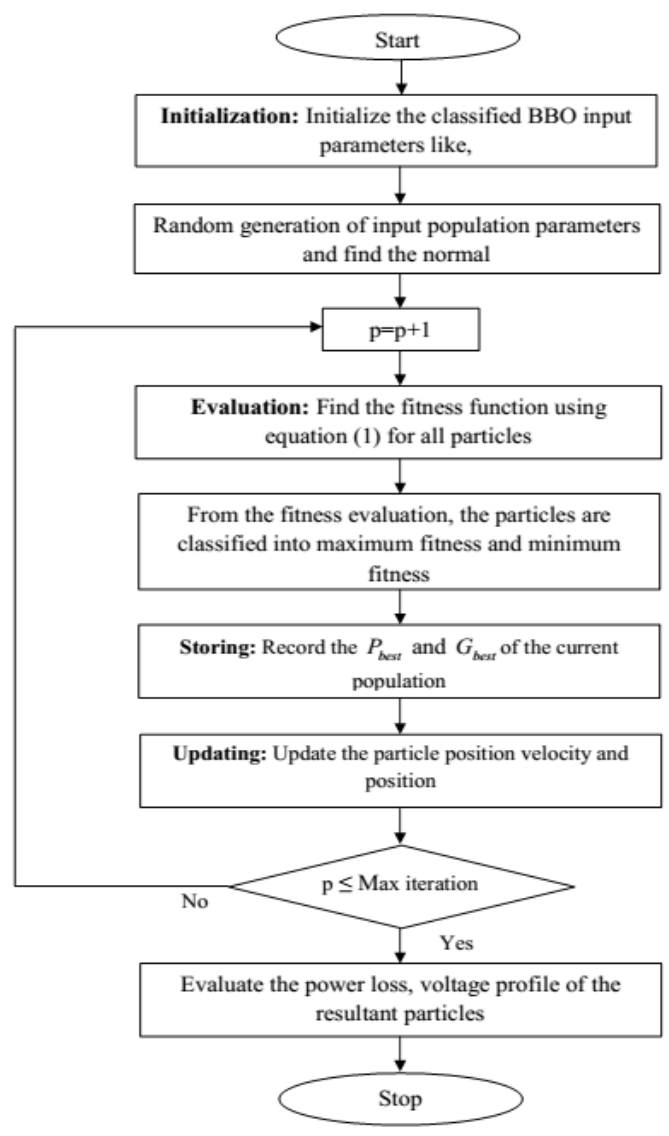

Fig. 4. Structure of the PSO based optimization as the DG sources. The suitable placement of wind and PV systems are determined and their capacity also evaluated. Here, the test case analysis is done by using IEEE standard bench mark 33 bus system. The IEEE standard bench mark system 33 bus system is utilized for the optimum location and sizing of the DG analysis. The effectiveness of the proposed method is identified by using the comparative analysis with the BBO and PSO technique. Here, the minimization of power loss, voltage deviation and load balancing are considered as the objective function. The proposed technique finds the optimum location and capacity of the DG depending on the objective function. The validation of the proposed method by using the IEEE 33 radial distribution system is described in the following section 5.1 .

\subsection{Validation of IEEE 33 bus distribution systems}

This section describes the proposed method validation using the proposed method. Here, the tested distribution system structure is shown in Fig. 5, in which 33 nodes, 32 branches [33]. The evaluations are carried out in the pu system with $12.66 \mathrm{kV}$ and 100MVA. The IEEE 33 bus radial distribution system structure is described in Fig. 3. Here, the IEEE 33 bus radial distribution system voltage, power loss evaluations are done by the proposed method. The results are displayed by the following figures.

Initially, the ANN method is utilized for analyzing the capacity of the DG sources with in the 24 hours. In each lhour, the minimum and maximum allowable limits are

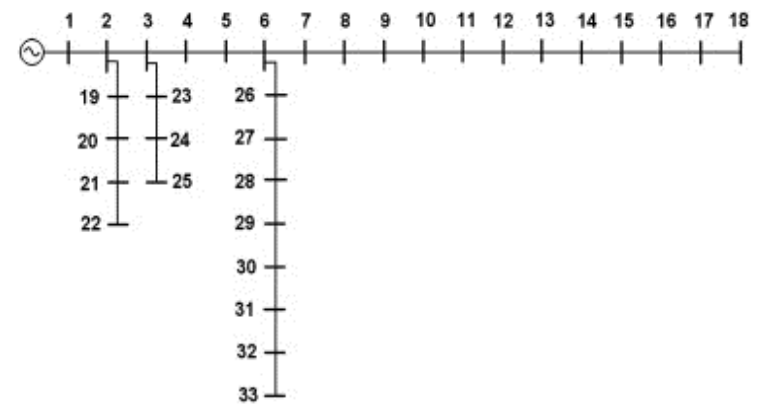

Fig. 5. Structure of the IEEE 33 bus distribution system

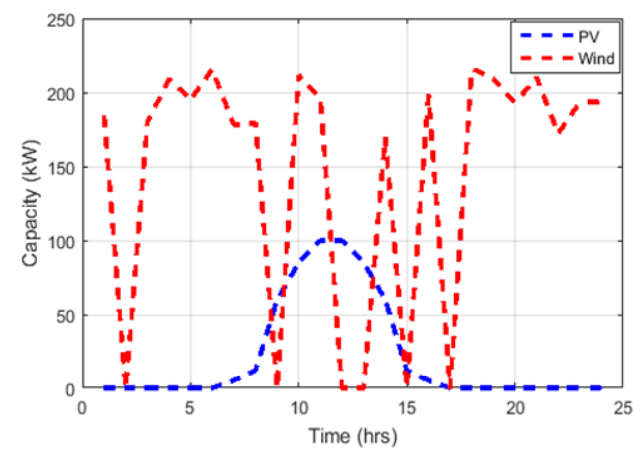

Fig. 6. Capacity analysis of DG sources 
determined from the ANN technique for the wind and PV sysetms. Fig. 6 represents the DG capacity analysis for 24hours, which represents the availability of wind and PV systems. After that, the votlage, power and power loss are evaluated under normal and faulty periods. The performance of ANN training and validation is illustrated in the Figs. 7 and 8.

The following Fig. 9 (a) and (b) describes the IEEE 33 bus radial distribution system normal voltage profile. It was seen that the radial distribution network maintains the bus voltage within the specified limit $(0.95$ to $1.05 \mathrm{pu})$ at all the 33 buses. The Fig. 10 shows that the bus voltage of the IEEE 33 bus radial distribution system at fault time. Due to the load increasing of the bus in the distribution system the bus voltage is violated from the normal condition. Here, the faults are analyzed in different five locations, which are illustrated in the following Figs. 11. It was seen that the load fault is diagnosis by using the specified capacity of DG sources like wind and PV. The

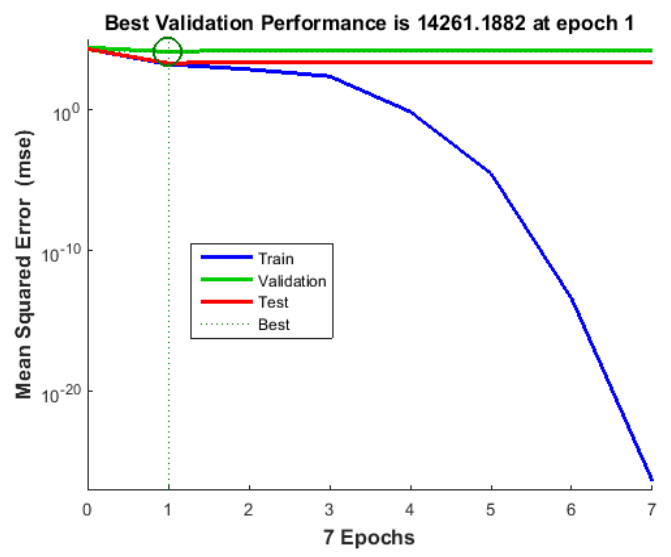

Fig. 7. Performance analysis of ANN
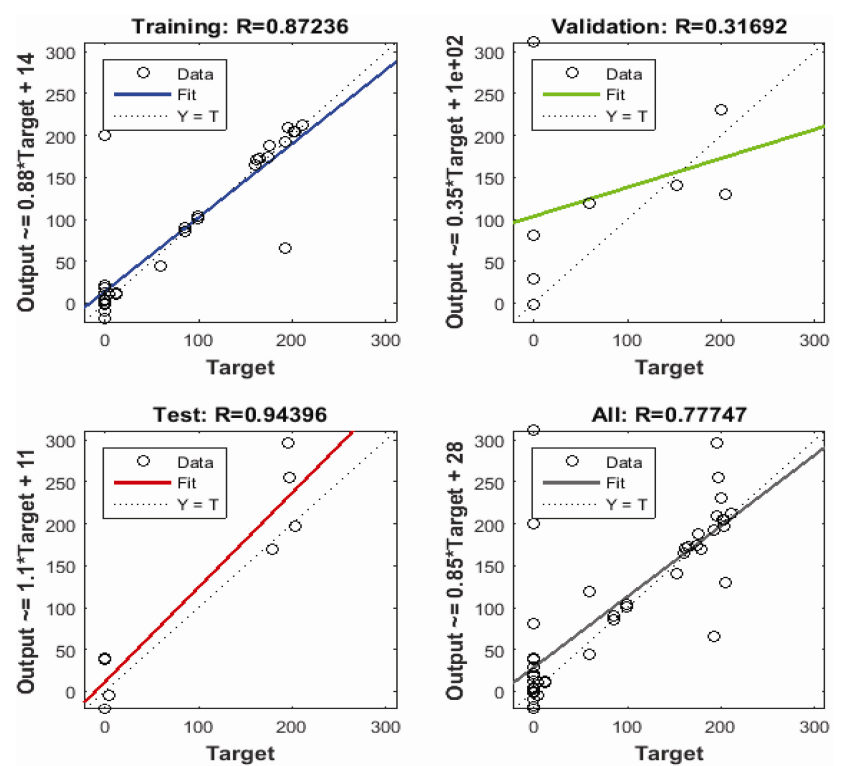

Fig. 8. Performance analysis of ANN training and validation
DG location and capacity is identified by the proposed technique. Here, the voltage profile of the IEEE 33 bus radial distribution system contains variations from the normal voltage profile. Then the load fault situation is evaluated using the proposed technique, which is explained in the following figures.

The IEEE 33 bus distribution system power loss is evaluated at the normal condition. The maximum power loss of the IEEE 33 bus distribution system at normal condition is 210 . In the figure, it was clearly shown that the many number of buses have less than $10 \mathrm{~kW}$ losses. During the loading period, the power loss of the IEEE 33 bus radial distribution system is analyzed and compared with the proposed method. It was seen that the power loss is increased due to the load variation of the distribution system. In the fault condition the bus power loss could be increased at 220 , it is a high loss compared to the normal condition. The IEEE 33 bus radial distribution system fault condition is diagnosed by using different techniques and described in the Fig. 10. From the figure we identified that the PSO could not effectively reduce the power loss of the distribution system.

Based on the objective function, the fitness is evaluated using proposed and existing methods. The illustration of the fitness function is represented in the Fig. 11, which shows that the proposed method achieved better results compared with other existing techniques.

Fig. 12 describes the convergence characteristic of objective function comparison, which takes 100 numbers

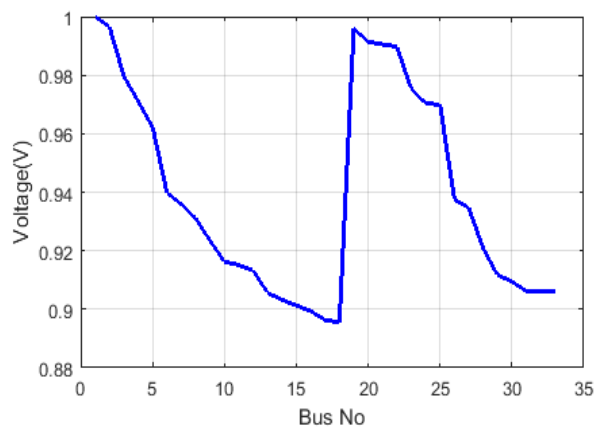

(a)



(b)

Fig. 9. Analysis of (a) voltage and (b) power in normal condition 


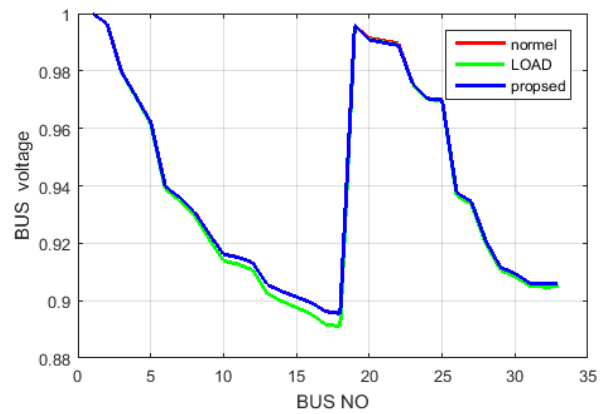

(a)



(c)

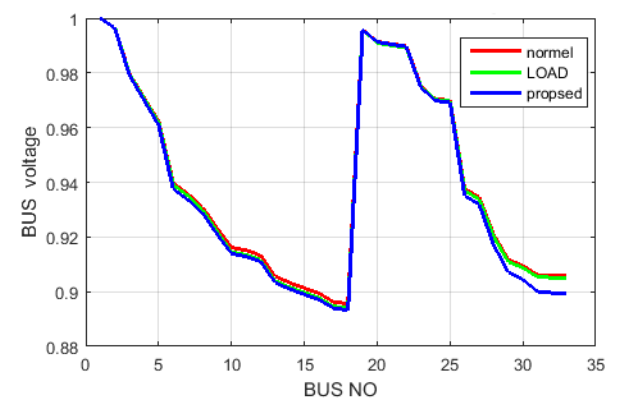

(b)

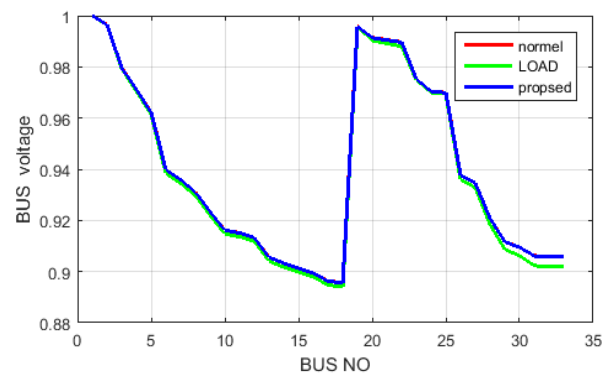

(d)

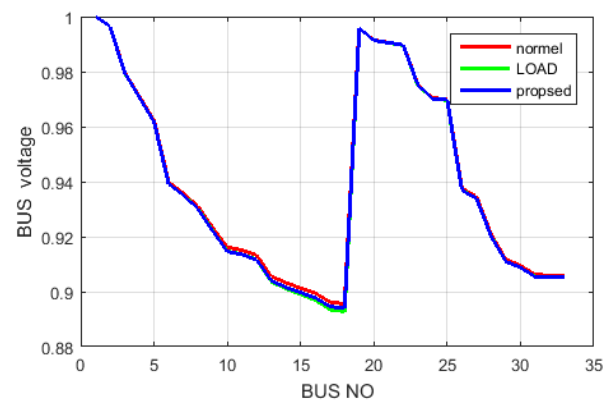

(e)

Fig. 10. Analysis of fault in five different locations using proposed methods

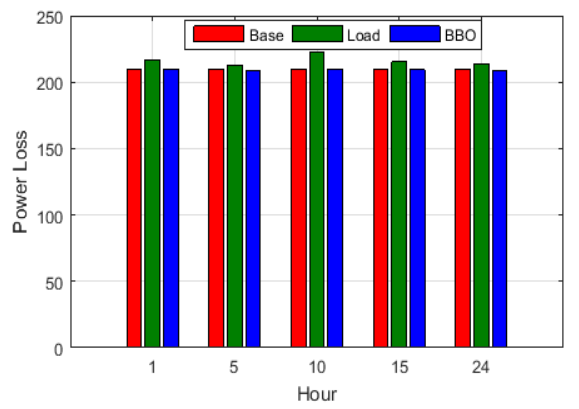

(a)

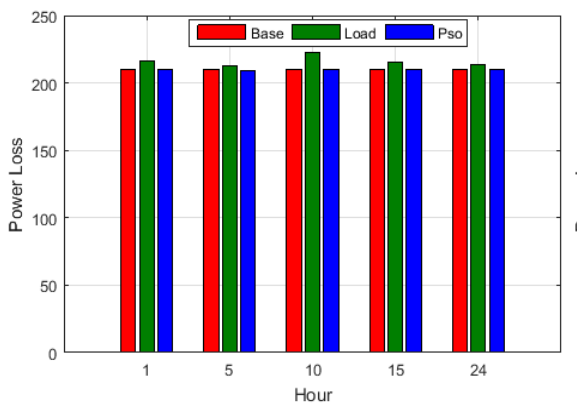

(b)

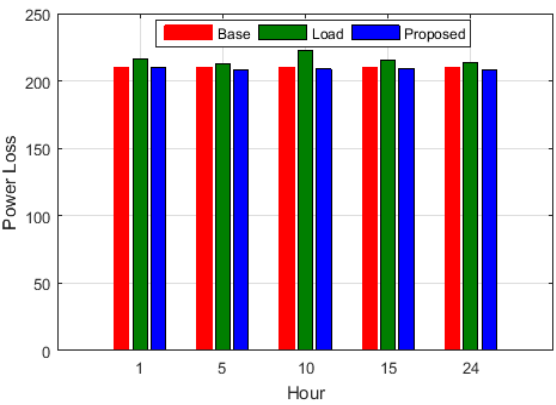

(c)

Fig. 11. Analysis of power loss using (a) BBO method (b) PSO and (c) proposed method

of iteration for all the techniques. It shows the objective function evaluation by using the proposed technique, PSO and BBO method. From the figure we observe that the PSO makes high values during the iterations, which starts from 2.15 and finishes at 2. Similarly, the BBO method is analyzed. But the proposed technique takes lot of classification of input parameters, so it determines the objective function effectively, i.e., initial iteration condition the objective function is 1; it is gradually reduced according to the variation of the number of iterations and at the end of the 100 iteration the objective function is 0.66 . The comparison analysis of the voltage and power loss is 


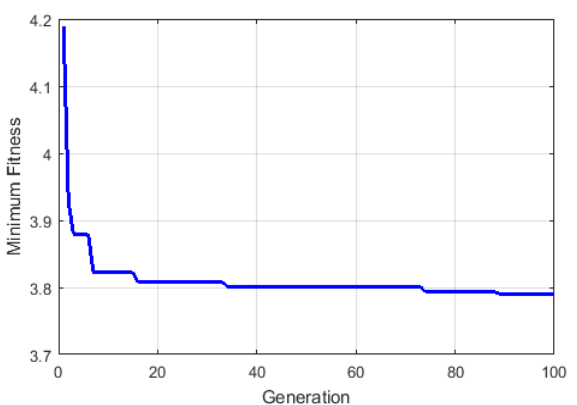

(a)

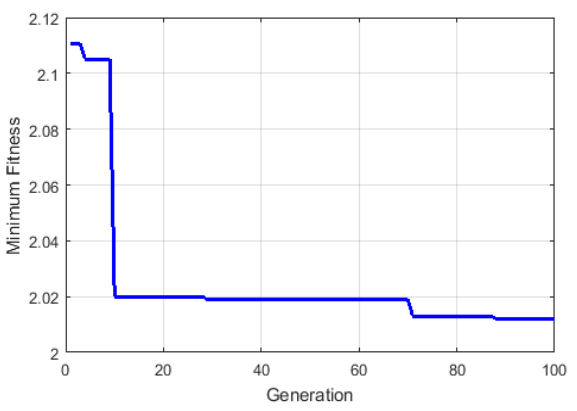

(b)

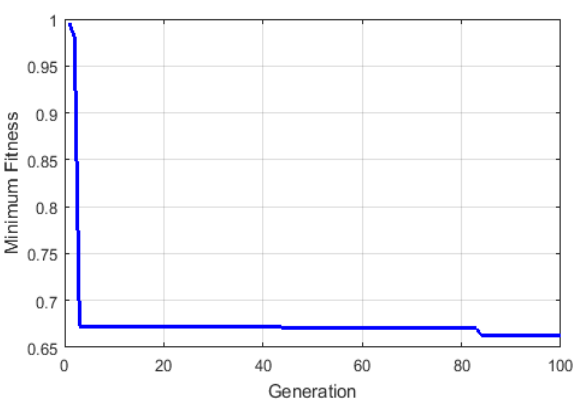

(c)

Fig. 12. Analysis of fitness function using (a) BBO (b) PSO and Proposed method

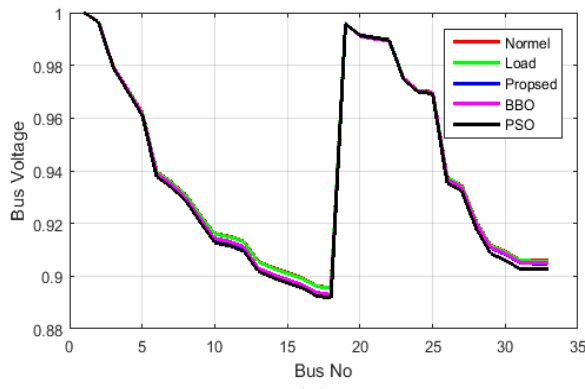

(a)

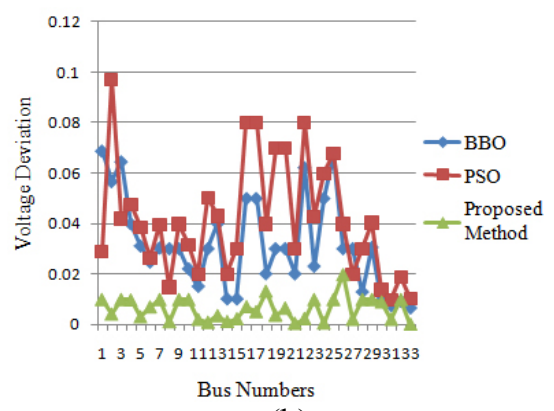

(b)



(c)

Fig. 13. Comparison Analysis of (a) voltage (b) voltage deviation and (c) power loss

described in the following section.

\subsection{Comparison analysis}

The bus voltage, voltage deviation and power loss of the radial distribution system is described in the Fig. 13. In the figure the radial distribution system bus voltage is analyzed with the BBO, PSO and the proposed technique. Initially the radial distribution system normal power flow is determined and then the fault may be the load increasing fault introduced in the system. Due to the load fault, the power loss of the bus system is violated; it is resolved by adding the optimum range of DG sources (wind and PV) at the specified location. In the figure the bus voltage profile using PSO is highly deviated compared to the proposed method and the BBO also cannot regulate the bus voltage at specified range. But the proposed method effectively maintains the bus voltage profile near at the $1 \mathrm{pu}$ range. From the analysis of voltage deviation, the proposed method maintains the minimum deviation compared with the other methods. The overall analysis of the proposed method gives better results compared with the existing technique.

\section{Conclusion}

This paper explains a proposed technique is utilized for detecting the location and the capacity of DG sources in power system. Here, the wind and PV are considered as the DG sources. The test case is done by using IEEE bench mark 33 bus system. Initially, the availability of these sources is analyzed in the 24 hours using ANN technique. After that, the voltage, power and power loss are analyzed in the normal and faulty conditions. In the proposed method, the optimal location and capacity of the DG sources is initially determined by using $\mathrm{BBO}$, then the $\mathrm{BBO}$ input parameters are classified into the sub parameters and allowed as the PSO input. By using the sub parameters the PSO finds the optimum location and capacity of DG. Finally the best results were taken among the techniques. This process is tested in the IEEE standard radial distribution bench mark system and the effectiveness is analyzed with the BBO and PSO algorithm. Here the comparison takes the radial distribution system power loss, voltage at various conditions like normal condition, during the fault time, BBO technique and proposed technique. From the comparison results we finalized that the proposed method is the well effective technique to identify the optimum location and capacity of the DG for the radial distribution system, which is competent over the other techniques.

\section{References}

[1] Duong Quoc Hung, N Mithulananthan and R. C. Bansal, "Analytical strategies for renewable distri- 
buted generation integration considering energy loss minimization”, Applied Energy, Vol. 105, pp. 75-85, 2013

[2] Sh Abdi and K Afshar, "Application of IPSO-Monte Carlo for optimal distributed generation allocation and sizing", Electrical Power and Energy Systems, Vol. 44, pp. 786-797, 2013

[3] Wen-Shan Tan, Mohammad Yusri Hassann, Md Shah Majid and Hasimah Abdul Rahman, "Optimal distributed renewable generation planning: A review of different approaches", Renewable and Sustainable Energy Reviews, Vol. 18, pp. 626-645, 2013

[4] Carmen L T Borges and Djalma M Falca o, "Optimal distributed generation allocation for reliability, losses, and voltage improvement", Electrical Power and Energy Systems, Vol. 28, pp. 413-420, 2006

[5] Rene Prenc, Davor skrlec and Vitomir Komen, "Distributed generation allocation based on average daily load and power production curves", Electrical Power and Energy Systems, Vol. 53, pp. 612-622, 2013

[6] J. C. Hernandez, F. J. Ruiz-Rodriguez and F. Jurado, "Technical impact of photovoltaic-distributed generation on radial distribution systems: Stochastic simulations for a feeder in Spain", Electrical Power and Energy Systems, Vol. 50, pp. 25-32, 2013

[7] S Devi and M Geethanjali, "Application of Modified Bacterial Foraging Optimization algorithm for optimal placement and sizing of Distributed Generation", Expert Systems with Applications, pp. 1-10, 2013

[8] Carmen Lucia Tancredo Borges, "An overview of reliability models and methods for distribution systems with renewable energy distributed generation", Renewable and Sustainable Energy Reviews, Vol. 16, pp. 4008-4015, 2012

[9] Rajkumar Viral and D K Khatod, "Optimal planning of distributed generation systems in distribution system: A review", Renewable and Sustainable Energy Reviews, Vol. 16, pp. 5146-5165, 2012

[10] Faruk Ugran and Engin Karatepe, "Multiple-distributed generation planning under load uncertainty and different penetration levels", Electrical Power and Energy Systems, Vol. 46, pp. 132-144, 2013

[11] A M El-Zonkoly, "Optimal placement of multidistributed generation units including different load models using particle swarm optimization", Swarm and Evolutionary Computation, Vol. 1, pp. 50-59, 2011

[12] MA Junjie, WANG Yulong and LIU Yang, "Size and Location of Distributed Generation in Distribution System Based on Immune Algorithm", Systems Engineering Procedia, Vol. 4, pp. 124-132, 2012

[13] Florina Rotaru, Gianfranco Chicco, Gheorghe Grigoras and Gheorghe Cartina, "Two-stage distributed generation optimal sizing with clustering-based node selection”, Electrical Power and Energy Systems, Vol.
40, pp. 120-129, 2012

[14] Sudipta Ghosh, S P Ghoshal and Saradindu Ghosh, "Optimal sizing and placement of distributed generation in a network system", Electrical Power and Energy Systems, Vol. 32, pp. 849-856, 2010

[15] Augusto C Rueda-Medina, John F Franco, Marcos J Rider, Antonio Padilha-Feltrin and Rubén Romero, "A mixed-integer linear programming approach for optimal type, size and allocation of distributed generation in radial distribution systems", Electric Power Systems Research, Vol. 97, pp. 133-143, 2013

[16] M R AlRashidi and M F AlHajri, "Optimal planning of multiple distributed generation sources in distribution networks: A new approach", Energy Conversion and Management, Vol. 52, pp. 3301-3308, 2011

[17] A R R de Souza, T S P Fernandes, A R Aoki, M R Sans, A P Oening, D C Marcilio and J S Omori, "Sensitivity analysis to connect distributed generation", Electrical Power and Energy Systems, Vol. 46, pp. 145-152, 2013

[18] A Shahmohammadi and M T Ameli, "Proper sizing and placement of distributed power generation aids the intentional islanding process", Electric Power Systems Research, Vol. 106, pp. 73-85, 2014

[19] Qi Kang, Tian Lan, Yong Yan, Lei Wang and Qidi $\mathrm{Wu}$, "Group search optimizer based optimal location and capacity of distributed generations", Neurocomputing, Vol. 78, pp. 55-63, 2012

[20] Juan Andrés Martín García and Antonio José Gil Mena, "Optimal distributed generation location and size using a modified teaching-learning based optimization algorithm", Electrical Power and Energy Systems, Vol. 50, pp. 65-75, 2013

[21] Partha Kayal and C.K. Chanda, "Placement of wind and solar based DGs in distribution system for power loss minimization and voltage stability improvement", International Journal of Electrical Power \& Energy Systems", Vol. 53, pp. 795-809, December 2013

[22] M. M. Aman, G. B. Jasmon, A. H. A. Bakar and H. Mokhlis, "A new approach for optimum simultaneous multi-DG distributed generation Units placement and sizing based on maximization of system loadability using HPSO (hybrid particle swarm optimization) algorithm", Energy, Vol. 66, No.1, pp. 202-215, March 2014

[23] Zhaoyu Wang, Bokan Chen, Jianhui Wang and Jinho Kim, "Robust Optimization Based Optimal DG Placement in Microgrids", IEEE Transactions on Smart Grid, Vol. 5, No. 5, pp. 2173-2182, 2014.

[24] Srinivasa Rao Gampa and D. Das, "Optimum placement and sizing of DGs considering average hourly variations of load", International Journal of Electrical Power \& Energy Systems, Vol. 66, pp. 2540, March 2015

[25] Ke-yan Liu, Wanxing Sheng, Yuan Liu, Xiaoli Meng 
and Yongmei Liu, "Optimal sitting and sizing of DGs in distribution system considering time sequence characteristics of loads and DGs", International Journal of Electrical Power \& Energy Systems, Vol. 69, pp. 430-440, 2015.

[26] Rajkumar Viral and D.K. Khatod, "An analytical approach for sizing and siting of DGs in balanced radial distribution networks for loss minimization", International Journal of Electrical Power \& Energy Systems, Vol. 67, pp. 191-201, 2015.

[27] Partha Kayal and C.K. Chanda, "Optimal mix of solar and wind distributed generations considering performance improvement of electrical distribution network", Renewable Energy, Vol. 75, pp. 173-186, 2015.

[28] Bretas, A. S. and Phadke, A. G., "Artificial neural networks in power system restoration", IEEE Transactions on Power Delivery, Vol. 18, No. 4, pp. 11811186, 2003.

[29] Bhattacharya A. and Chattopadhyay PK, "Biogeography-based optimization for solution of optimal power flow problem", In proceedings of IEEE conference on Electrical Engineering/Electronics Computer Telecommunications and Information Technology, pp. 435-439, 2010

[30] Li J and Zhao J., "Combining Differential Evolution Algorithm with biogeography-based optimization algorithm for reconfiguration of distribution network", In proceedings of IEEE conference on Power System Technology, pp. 1-6, 2012.

[31] Li X, Liu YS and Yang SX, “Application of improved PSO to power transmission congestion management optimization model", Journal of Central South University of Technology, pp. 347-51, 2008

[32] Pappala VS and Erlich I., "Power system optimization under uncertainties: A PSO approach", In proceedings of IEEE Symposium on Swarm Intelligence, pp. 1-8, 2008.

[33] Peter Musau Moses, Nicodemus Abungu Odero and Mwangi Mbuthia, "Reducing Real and Reactive Power Losses in the Power Distribution System by DFIG Placement and Sizing Using Ordinary PSO and HGAPSO: A Comparison", International Journal of Emerging Technology and Advanced Engineering, Vol. 2, No. 11, November 2012.

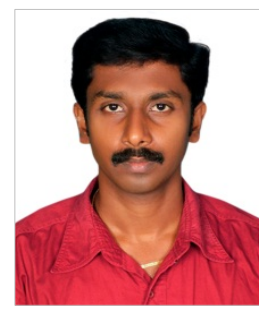

M. Durairasan, B.E., M.E., is working as Assistant Professor in University College of Engineering, Thirukkuvalai, Nagapattinam District in Tamilnadu, India who has over twelve years of experience in teaching. He has completed B.E Degree in Electrical and Electronics Engineering (EEE) in
Adhiparasakthi Engineering College, Melmaruvathur in 2000; M.E. Process Control and Instrumentation degree in Annamalai University, in 2004. He has attended several National and International conferences and workshops. He is a life member of Indian Society for Technical Education (ISTE).His specialization is Hybrid renewable Energy systems, power system optimization and Distributed generation.

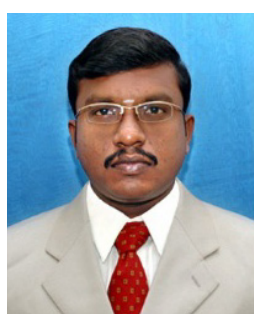

A. Kalaiselvan received the B.Sc., M.Sc., and M. Phil degrees from Bharathidasan University, Tiruchirappalli, Tamilnadu, INDIA and the Ph.D. degree from the same Bharathidasan University, Tiruchirappalli, Tamilnadu, INDIA in 2006. From 2007-2008, he did his post-doctoral research at CEA, Grenoble, France. He is currently working as an Assistant Professor in the Department of Chemistry at University College of Engineering (A Constitutent College of Anna University Chennai). His specialization is Computational Chemistry and his research interests are modeling reactions by characterizing transition states, intermediates and complexes and Hybrid Energy Systems.

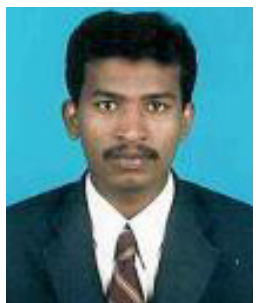

H. Habeebullah Sait was born in Tamilnadu, India in 1979. He received the B.E degree in electrical engineering from Syed Ammal Engg College (SAEC), Ramnad, Madurai Kamaraj University (MKU), Tamilnadu, India in 2002. He received the M.E degree in Power Electronics \& Drives from MEPCO SCHLENK Engg College, Sivakasi, Anna University, Tamilnadu India in 2005 and Ph.D. degree in Power electronics from National Institute of Technology, Tiruchirappalli, Tamilnadu, India in 2010. His areas of interests are application of power electronics in renewable energy systems, Power Quality, FACTS and Multi level Inverter. 